\section{UCSC Genome Browser}

J. Arnemann

Abteilung Molekulargenetik, Labor Dr. Wisplinghoff, Köln, Deutschland

Synonym(e) Genombrowser

Englischer Begriff UCSC Genome Browser

Definition Der UCSC Genome Browser (https://genome. https://genome.ucsc.edu ucsc.edu) ist eine interaktive Website zu Genomanalysen.
Beschreibung Der UCSC Genome Browser wird von der University of California Santa Cruz (UCSC) als Host geführt. Er ermöglicht den Zugang zu genomischen Sequenzdaten, zur schnellen Visualisierung von Analysen, zu Suchanfragen und zu interaktiven Auswertungen von Forschungsaspekten zum Genom. Der UCSC Browser ist als Open Source frei zugänglich.

\section{Literatur}

